\title{
Scombroid poisoning
}

\author{
Piero Stratta MD, Guido Badino PhD
}

\section{Scombroid poisoning is a common form of food poisoning related to fish ingestion}

Scombroid poisoning occurs after the ingestion of fresh, canned or smoked fish with high histamine levels due to improper processing or storage. First described in conjunction with fish in the suborder Scombroidea (e.g., tuna and mackerel), it has since been described with other dark-fleshed fish (e.g., sardines and anchovies). Scombroid poisoning is one of the most common causes of morbidity associated with fish intake. ${ }^{1,2}$

\section{Symptoms are that of histamine poisoning}

Symptoms of scombroid poisoning include flushing, rash, urticaria (generally widespread erythema, usually lacking wheals), palpitations, headache, dizziness, sweating, and burning of the mouth and throat. Gastrointestinal symptoms can include abdominal cramps, nausea, vomiting and diarrhea. Bronchospasm, respiratory distress and vasodilatory shock have also been described. Symptoms begin within 10 to 90 minutes after eating the implicated fish. The rash lasts 2-5 hours, and the other symptoms usually disappear within 3-36 hours. The diagnosis is often clinical and can be confirmed by measurement of histamine in incriminated spoiled food. Determination of the patient's histamine level in plasma or the level of histamine metabolites (e.g., $N$-methylhistamine) in urine can also support the diagnosis. ${ }^{4}$

\section{Scombroid poisoning is not an allergy}

Allergic reactions typically affect one person who has a history of allergy to the implicated food item. However, with scombroid poisoning, more than one person, often with no history of allergies, may be affected. ${ }^{3}$ Histamine produced by the decarboxylation of histidine in the muscle of the fish is primarily responsible for the condition. The decarboxylation process is induced by enzymes produced by primarily enteric gram-negative bacteria (e.g., Morganella morganii, Escherichia coli, Klebsiella species and Pseudomonas aeruginosa) found in the fish's cutis and intestines. ${ }^{1,2}$

\section{Histamine does not change the smell or appearance of the affected fish}

Scombroid poisoning is frequently misdiagnosed. ${ }^{5}$ Because histamine does not alter the organoleptic quality, the fish may seem normal. However, elevated histamine levels can occur in fish owing to improper refrigeration before processing or to storage of the fish at room temperature after cooking. Therefore, the appearance, taste and smell of the fish are poor guides as to the presence of histamine. Histamine is heat-stable and remains present after cooking, freezing, canning or smoking. Outbreaks are most common in summer. ${ }^{1,2}$

\section{References}

1. Jantschitsch C, Kinaciyan T, Manafi M, et al. Severe scombroid fish poisoning: an underrecognized dermatologic emergency. J Am Acad Dermatol 2011; 65:246-7.

2. Hungerford JM. Scombroid poisoning: a review. Toxicon 2010;56:231-43.

3. O'Connor MM, Forbes GM. Scombroid poisoning: not fish allergy. Aust N Z J Med 2000;30:520.

4. Morrow JD, Margolies GR, Rowland J, et al. Evidence that histamine is the causative toxin of scombroid-fish poisoning. N Engl J Med 1991;324:716-20.

5. Attaran RR, Probst F. Histamine fish poisoning: a common but frequently misdiagnosed condition. Emerg Med J 2002;19:474-5.
Treatment involves antihistamines and supportive care

Most instances of scombroid poisoning are self-limited. However, if the patient has symptoms severe enough to require treatment, rapid-acting antihistamines (usually $\mathrm{H}_{1}$-receptor antagonists) are commonly used, along with supportive care tailored to the symptoms. Adrenalin and corticosteroids are generally not indicated. ${ }^{1,25}$ To prevent further instances of poisoning, public health authorities should be notified to investigate the source and remove the implicated product from distribution.

Competing interests: None declared.

This article has been peer reviewed.

Affiliations: From the Department of Nephrology and Transplantation (Stratta), Amedeo Avogadro University, Novara, Italy; and the Department of Animal and Human Biology (Badino), University of Torino, Torino, Italy

Correspondence to: Dr. Piero Stratta, piero.stratta @med.unipmn.it

CMAJ 2012. DOI:10.1503/cmaj.111031 